\title{
WHO News and activities
}

\section{Risk from radon gas indoors ${ }^{a}$}

In 1979 the WHO Regional Office for Europe (EURO) drew the attention of national health authorities in European countries to the dangers posed by radon as a pollutant in indoor atmospheres. On the basis of a 1982 report by the United Nations Scientific Committee on the Effects of Atomic Radiation (UNSCEAR), it was subsequently estimated that an annual incidence of 10-40 cases of lung cancer per million persons would be expected to arise from exposure to a mean indoor radon progeny concentration of $15 \mathrm{~Bq} / \mathrm{m}^{3}$. Use of the relative-risk model indicated that about $5-15 \%$ of the observed lung cancer incidence or of the lifetime risk of lung cancer may be attributed to indoor radon progenies. In several European countries, the proportion of individuals living in dwellings with greater than $100 \mathrm{~Bq} / \mathrm{m}^{3}$ equilibrium equivalent radon (EER) concentrations could be as high as $10 \%$, for example, in Scandinavia. In 1985, a EURO working group recommended that simple remedial measures should be considered for buildings with average annual radon progeny levels greater than $100 \mathrm{~Bq} / \mathrm{m}^{3} \mathrm{EER}$.

By 1990 several countries had introduced national regulations restricting levels of radon progeny in buildings. For example, in Sweden requirements were set limiting the levels of radon progeny in new buildings to $70 \mathrm{~Bq} / \mathrm{m}^{3}$ EER and aiming at an average of $25 \mathrm{~Bq} / \mathrm{m}^{3}$ EER.

In 1991 at a conference organized by the International Commission for Radiological Protection (ICRP) concern was expressed that there were no universally accepted guidelines and regulations for the control of the levels of radon and its progeny in buildings. It was also agreed that the risk posed by radon as a source of radiation should be evaluated and controlled in a similar way to the risks from other radiation sources and that the principle of "as low as reasonably attainable" should prevail.

Since the socioeconomic factors involved in dealing with radon in buildings may be important, new approaches are needed to the problem. Although the concentrations of radon and its progenies should in theory be set to zero, this is not possible because of their natural background levels (for radon, the level ranges from $0.1 \mathrm{~Bq} / \mathrm{m}^{3}$ over oceans to 10 $\mathrm{Bq} / \mathrm{m}^{3}$ over land, with an average of $3 \mathrm{~Bq} / \mathrm{m}^{3}$ at ground level). Moreover, the effects of condemning private homes and other buildings, e.g., schools, hos-

\footnotetext{
a Based on an article by $M$. Suess, until recently Adviser for Environmental Health Hazards, WHO Regional Office for Europe, Copenhagen, Denmark.

Reprint No. 5480
}

pitals, and offices, can be considerable. Hence, instead of using the existing guideline concentration values for radon and its progeny, which appear to inhibit making improvements to the situation in many countries, newly established health criteria are needed to determine the resulting health risk in buildings. This would permit national and local authorities to have greater flexibility in enacting control measures, giving consideration, for example, to the number of people directly affected, the financial implications to such individuals, the authorities, and the community, as well as to the overall risk the population is willing to accept and maintain. Furthermore, the risk to the population from radon could then be weighed up against that from other factors (including tobacco smoke), thereby facilitating comparison of the financial implications.

\section{Tuberculosis preventive therapy in HIV-infected individuals ${ }^{b}$}

This statement is intended to assist health care providers in considering the use of isoniazid preventive therapy in persons with human immunodeficiency virus (HIV) infection. Currently there is insufficient information to recommend that tuberculosis preventive therapy for co-infected persons be implemented as one of the components of the tuberculosis control strategy in programme settings worldwide. This awaits more detailed information on the efficacy of preventive therapy and, in particular, confirmation of its cost-effectiveness and operational feasibility. It must be emphasized that the primary objective of a tuberculosis programme is the interruption of tuberculosis transmission by the curative treatment of infectious tuberculosis cases. Thus, programme implementation of preventive therapy would only be considered in areas where tuberculosis programmes are achieving the global targets established by WHO (i.e., successfully treating $85 \%$ of detected smearpositive cases and detecting $70 \%$ of existing new smear-positive cases) and where voluntary testing and counselling for HIV infection is available. This will also require close collaboration between national acquired immunodeficiency syndrome (AIDS) and tuberculosis control programmes.

It is now well established that infection with HIV is the most potent factor that promotes the

\footnotetext{
b Based on: Tuberculosis preventive therapy in HIV-infected individuals: a Joint Statement of the WHO Tuberculosis Programme and the Global Programme on AIDS and the International Union against Tuberculosis and Lung Disease (IUATLD). Weekly epidemiological record, 1993, 68(49): 361-364.
} 
development of tuberculosis in individuals infected with Mycobacterium tuberculosis. The estimated annual risk of tuberculosis in persons with HIV and tuberculosis co-infection is $5-10 \%$, with a lifetime risk of $50 \%$ or greater. In areas where both HIV infection and tuberculosis are common, notably in countries in sub-Saharan Africa, HIV has had a tremendous impact on the tuberculosis situation.

A large number of placebo-control clinical trials in tuberculin-positive persons at increased risk of tuberculosis have shown that daily administration of isoniazid for periods of 6-12 months substantially reduces the risk of tuberculosis. In areas with low annual rates of tuberculosis infection this protection is probably lifelong. Current International Union against Tuberculosis and Lung Disease (IUATLD) guidelines on tuberculosis in children recommend isoniazid preventive therapy for childhood contacts of infectious cases. The same recommendation has been made by WHO.

Available information suggests that isoniazid preventive therapy is also effective in preventing tuberculosis in individuals with HIV infection. Thus, preventive therapy is the single intervention that is both readily available and affordable and which might reduce the occurrence of $\mathrm{HIV}$-associated tuberculosis in co-infected persons. Moreover, preliminary observations suggest that tuberculosis may increase progression of HIV infection and that tuberculosis preventive chemotherapy may offer the possibility of prolonging the survival of HIV-infected persons.

Thus, tuberculosis preventive therapy with isoniazid may be indicated for an HIV-seropositive person with a positive tuberculin test as an individual health measure, if the subject is free of active tuberculosis. To administer preventive therapy safely, it is mandatory that appropriate facilities to screen individuals for active tuberculosis are available and that patients are monitored during therapy. The minimal requirements for screening are a chest radiograph and acid-fast microscopy. Facilities for culturing specimens for mycobacteria are also desirable.

\section{Screening for tuberculosis and tuberculosis infection}

Education about tuberculosis and its link to HIV infection should be a part of HIV/AIDS pre- and post-test counselling. Individuals who are found to be HIV seropositive should be screened for tuberculosis by clinical examination. Those being considered for tuberculosis preventive therapy should receive an intradermal tuberculin skin test (Mantoux test) with a product equivalent to 5 TU of PPD-S or 2 TU of PPD-RT23. A positive test indicating tuber- culosis infection is defined as a reaction of at least $5 \mathrm{~mm}$ induration after 48-72 hours. Although BCG vaccination induces reactivity to $P P D$, the $5 \mathrm{~mm}$ cutoff for a positive skin test is appropriate for adults with HIV infection regardless of history of vaccination.

It is critically important to exclude the presence of active tuberculosis before beginning preventive therapy. Therefore, those persons with a positive tuberculin skin test should have a chest radiograph. Sputum samples should be collected for bacteriological examination also from persons with symptoms compatible with pulmonary tuberculosis and those with abnormal chest radiographs. Those with signs and symptoms consistent with extrapulmonary tuberculosis (e.g., peripheral lymphadenopathy) should be appropriately investigated.

HIV-positive persons with negative tuberculin skin tests living in areas endemic for tuberculosis may be reassessed periodically (e.g., every 6 months) and managed accordingly. Anergy to tuberculin is commonly found in persons with HIV infection, especially in the advanced stages of immunosuppression. Although there are only limited data supporting isoniazid preventive therapy for anergic persons at risk of tuberculosis, some authorities have recommended this practice. However, the administration of isoniazid to HIV-infected persons without tuberculin testing has not been evaluated and cannot currently be recommended.

\section{Administration of isoniazid preventive therapy}

Isoniazid preventive therapy is indicated for tuberculin-positive, HIV-infected persons who do not have active tuberculosis. For preventive therapy in both children and adults, isoniazid, at a daily dose of $5 \mathrm{mg} / \mathrm{kg}$ up to a maximum of $300 \mathrm{mg}$, should be given for 6-12 months. Drug supplies should be issued at monthly intervals and patients assessed for compliance, drug toxicity, and signs of active tuberculosis. Isoniazid may be given twice weekly at a dose of $15 \mathrm{mg} / \mathrm{kg}$ for those requiring supervision, although there are no clinical studies demonstrating the efficacy of intermittent preventive therapy. Patients who interrupt treatment may be restarted with the aim of providing at least 6 months of preventive therapy over a year. Such persons should nevertheless not have preventive therapy restarted if they have developed signs and symptoms of tuberculosis and active tuberculosis has not been excluded.

Isoniazid-associated hepatotoxicity is the most important adverse consequence of isoniazid, occurring in up to $2 \%$ of persons over 50 years of age receiving the drug. Isoniazid preventive therapy is contraindicated in persons with chronic active hepatitis and should be given with caution to persons who 
consume alcohol daily. Although routine biochemical monitoring for hepatitis is not recommended, patients should be carefully educated about the signs and symptoms of hepatitis and instructed to discontinue the drug promptly should these occur.

\section{Alternative preventive therapy regimens}

Some authorities have recommended alternative preventive therapy regimens for persons intolerant of isoniazid and for those who are infected with isoniazid-resistant organisms, and there are limited clinical and experimental data supporting their use. However, their efficacy in preventing tuberculosis in persons with HIV infection has not yet been shown, and currently no alternative regimen to isoniazid is recommended for preventive therapy.

\section{Research issues}

There are a number of questions about tuberculosis preventive therapy in HIV infection which can only be answered by further studies. Among the more important of these are the following: the efficacy of "short-course" multidrug preventive therapy; the role of lifelong or extended preventive therapy in areas of high tuberculosis transmission and the role of preventive therapy for persons who are anergic. Also of paramount importance are operational studies to define the role of preventive therapy in programme conditions. Included in these studies should be a careful analysis of the feasibility of preventive therapy as measured by demand, sustainability, and costeffectiveness.

A list of references is available on request from the Tuberculosis Programme, Division of Communicable Diseases, World Health Organization, 1211 Geneva 27, Switzerland.

\section{Malaria chemoprophylactic regimens for travellers ${ }^{c}$}

Country-specific recommendations on chemoprophylactic regimens have been revised by WHO. A summary of these recommendations is outlined below, indicating areas for use and common side-effects of the drugs.

\section{Chloroquine}

Chloroquine is recommended for areas where Plasmodium vivax is the predominant species and for areas where $P$. falciparum is sensitive to chloroquine, including some parts of the Middle East and

\footnotetext{
- Based on: Malaria prophylaxis for travellers. Weekly epidemiological record, 1993, 68(51): 377-383.
}

South America. Chloroquine is administered as weekly prophylaxis at a dose of $300 \mathrm{mg}$ (base).

Chloroquine is usually well tolerated. The few people who do experience uncomfortable sideeffects, such as gastrointestinal disturbance, may tolerate it better by taking the drug with meals and in divided twice-weekly doses.

\section{Chloroquine plus proguanil}

Chloroquine plus proguanil is the recommended regimen for the following: areas where $P$. falciparum resistance to chloroquine has been reported, but is of low level, including some areas of South-East Asia and the Middle East; areas where $P$. falciparum highly resistant to chloroquine occurs, if the traveller cannot tolerate mefloquine or doxycycline; and areas where $P$. falciparum resistance is present but other drugs are contraindicated because of early pregnancy. This regimen consists of a dose of $300 \mathrm{mg}$ (base) of chloroquine taken weekly plus a dose of $200 \mathrm{mg}$ of proguanil each day.

Chloroquine plus proguanil often causes gastrointestinal upsets. In such an event, taking the drugs with meals, and dividing the chloroquine dosage into twice-weekly doses may reduce the side-effects.

\section{Mefloquine}

Mefloquine is recommended for areas where drugresistant $P$. falciparum is frequent and/or where high levels of resistance occur including some areas of south-east Asia, Indochina, and Africa. The drug is administered as weekly prophylaxis at a single dose of $250 \mathrm{mg}$.

Usually, mefloquine is well tolerated in persons with no contraindications. Mild side-effects such as dizziness or gastrointestinal effects may occur transiently during early prophylaxis, but they spontaneously resolve. Neurological and psychiatric disorders may rarely occur in persons with contraindications. Those experiencing such disorders should not take mefloquine again.

If mefloquine cannot be taken because it is contraindicated or is not tolerated, travellers should switch to either doxycycline or chloroquine plus proguanil. The latter has a lower efficacy in these areas.

\section{Doxycycline}

This drug is recommended for areas where multipledrug resistance is reported (e.g., Cambodia-Thai and Myanmar-Thai borders) and areas where highly resistant $P$. falciparum occurs and the traveller cannot tolerate mefloquine. The drug is administered as a single daily dose of $100 \mathrm{mg}$.

Travellers who use doxycycline should be cautioned about its side-effects, particularly skin sensitiv- 


\section{Notes et activités OMS}

\section{Risques liés à la présence de radon à l'intérieur des locaux ${ }^{a}$}

En 1979, le Bureau régional de l'OMS pour l'Europe (EURO) a attiré l'attention des autorités sanitaires des pays européens sur les risques liés à la présence de radon comme polluant de l'atmosphère de l'intérieur des locaux. Sur la base d'un rapport de 1982 émanant du Comité scientifique des Nations Unies pour l'étude des effets des rayonnements ionisants (UNSCEAR), il a été estimé qu'une incidence annuelle de 10 à 40 cas de cancers du poumon par million de personnes pourrait être due à l'exposition à une concentration moyenne de produits de filiation du radon à l'intérieur des locaux de $15 \mathrm{~Bq} / \mathrm{m}^{3}$. En utilisant un modèle de risque relatif, on trouve qu'environ 5 à $15 \%$ de l'incidence observée des cancers du poumon ou du risque de cancer du poumon sur une vie entière peuvent être attribués aux produits de filiation du radon à l'intérieur des locaux. Dans plusieurs pays européens, la proportion de personnes vivant dans des bâtiments où la concentration d'équivalent radon à l'équilibre (ERE) est supérieure à $100 \mathrm{~Bq} / \mathrm{m}^{3}$ pourrait atteindre $10 \%$, par exemple en Scandinavie. En 1985, un groupe de travail d'EURO a recommandé d'envisager d'adopter des mesures correctrices simples pour les bâtiments où les taux moyens annuels de produits de filiation du radon dépassent $100 \mathrm{~Bq} / \mathrm{m}^{3}$ en ERE.

En 1990, plusieurs pays avaient adopté une réglementation nationale limitant les taux de produits de filiation du radon dans les bâtiments. Par exemple, la Suède a établi des normes limitant les taux dans les nouveaux bâtiments à $70 \mathrm{~Bq} / \mathrm{m}^{3}$ en ERE, avec pour objectif d'atteindre une moyenne de $25 \mathrm{~Bq} / \mathrm{m}^{3}$ en ERE.

En 1991, lors d'une conférence organisée par la Commission internationale de Protection radiologique (CIPR), les participants se sont déclarés préoccupés du fait qu'il n'existait pas de directives universellement admises pour le contrôle des taux de radon et de ses produits de filiation dans les bâtiments. Il a également été convenu que le risque dû au radon en tant que source de rayonnement devait être évalué et contrôlé de la même façon que les risques résultant d'autres sources de rayonnement et qu'il fallait s'attacher au principe du taux "aussi bas qu'il est raisonnablement possible d'atteindre".

\footnotetext{
a D'après un article de $M$. Suess, Conseiller pour les risques pour la santé du fait de l'environnement, Bureau régional de l'OMS pour l'Europe, Copenhague, Danemark.

Tiré à part $\mathrm{N}^{\circ}: \mathbf{5 4 8 1}$
}

Comme les facteurs socio-économiques impliqués dans la prise en charge du problème du radon peuvent être importants, il est nécessaire de trouver de nouvelles approches. Bien qu'en théorie la concentration de radon et de ses produits de filiation doive être abaissée à zéro, cela n'est pas possible du fait du bruit de fond naturel (pour le radon, ce taux va de $0,1 \mathrm{~Bq} / \mathrm{m}^{3}$ au-dessus des océans à $10 \mathrm{~Bq} / \mathrm{m}^{3}$ au-dessus des terres émergées, avec une moyenne de $3 \mathrm{~Bq} / \mathrm{m}^{3}$ au niveau du sol). De plus, la condamnation de logements et d'autres bâtiments comme les écoles, les hôpitaux et les bureaux risque d'avoir des conséquences considérables. Par conséquent, au lieu d'utiliser les valeurs actuelles figurant dans les directives pour le radon et ses produits de filiation, qui empêchent en pratique de remédier à la situation dans nombre de pays, il faut établir de nouveaux critères, cette fois d'ordre sanitaire, pour déterminer le risque pour la santé découlant de la présence de radon et de ses produits de filiation dans les bâtiments. Il serait ainsi possible aux autorités nationales et locales de faire appliquer avec plus de souplesse les mesures de lutte, en tenant compte par exemple du nombre de personnes directement concernées, des conséquences financières pour ces personnes, pour les autorités et pour la communauté, ainsi que du risque général que la population est prête à accepter et à maintenir. De plus, le risque que le radon comporte pour la population pourrait être pesé par rapport aux risques découlant d'autres facteurs, notamment la fumée de tabac, ce qui faciliterait la comparaison des conséquences financières des mesures correctrices.

\section{Le traitement préventif de la tuberculose chez les sujets infectés par le VIH ${ }^{b}$}

La présente déclaration a pour but d'aider les dispensateurs de soins lorsqu'ils envisagent d'administrer de l'isoniazide à titre préventif aux sujets infectés par le virus de l'immunodéficience humaine (VIH). Les informations dont on dispose actuellement ne permettent pas de recommander que le traitement

\footnotetext{
b D'après: le traitement préventif de la tuberculose chez les sujets infectés par le $\mathrm{VIH}$ : Déclaration conjointe du programme de la tuberculose et du programme mondial de lutte contre le SIDA de l'OMS, et de l'Union internationale contre la Tuberculose et les Maladies respiratoires (UITMR). Relevé épidémiologique hebdomadaire, 1993, 68(49): 361-364.
} 
préventif de la tuberculose chez les sujets co-infectés soit une composante de la stratégie antituberculeuse dans les programmes mis en œuvre au niveau mondial. Il faudrait pour cela être mieux renseigné sur l'efficacité des traitements préventifs et, en particulier, avoir confirmation de leur rentabilité et de leur faisabilité pratique. Il faut insister sur le fait qu'un programme antituberculeux a pour principal objectif d'interrompre la transmission de la tuberculose par le traitement curatif des cas infectieux. C'est pourquoi la mise en cuvre de traitements préventifs dans le cadre d'un programme n'est à envisager que dans les régions où les programmes antituberculeux atteignent déjà les objectifs fixés à l'échelle mondiale par l'OMS (à savoir un taux de guérison de $85 \%$ des malades à frottis positifs dépistés et le dépistage de $70 \%$ des cas nouveaux à frottis positifs existants) et où l'on dispose de services de dépistage volontaire et de conseil anti-VIH. Cela demandera aussi une collaboration étroite entre les programmes nationaux de lutte contre le syndrome d'immunodéficience acquise (SIDA) et les programmes antituberculeux.

Il est maintenant bien établi que l'infection par le VIH est le facteur le plus propice au développement de la tuberculose chez les sujets infectés par Mycobacterium tuberculosis. Le risque de tuberculose chez les sujets infectés à la fois par le VIH et par le bacille tuberculeux est estimé à $5-10 \%$ par an et peut aller jusqu'à $50 \%$, voire plus, sur la durée d'une vie. Là où l'infection par le VIH et la tuberculose sont l'une et l'autre courantes, notamment dans les pays d'Afrique sub-saharienne, le VIH a eu un énorme impact sur le tableau de la tuberculose.

Un grand nombre d'essais cliniques contrôlés avec placebo effectués sur des sujets positifs au test à la tuberculine, qui sont exposés à un risque accru de tuberculose, ont fait apparaître que l'administration quotidienne d'isoniazide pendant des périodes de 6 à 12 mois réduit sensiblement le risque de tuberculose. Dans les régions où les taux annuels de tuberculoseinfection sont faibles, cette protection dure probablement toute la vie. Dans les grandes orientations qu'elle a publiées pour lutter contre la tuberculose chez l'enfant, l'Union internationale contre la Tuberculose et les Maladies respiratoires (UITMR) recommande d'administrer de l'isoniazide à titre préventif chez l'enfant en contact avec des cas infectieux. L'OMS a formulé la même recommandation.

Les informations disponibles donnent à penser que l'administration d'isoniazide à titre préventif est également efficace pour prévenir la tuberculose chez les sujets infectés par le VIH. Ainsi, le traitement préventif est la seule intervention à la fois facile à mettre en œuvre et d'un coût abordable qui pourrait restreindre l'apparition d'une tuberculose associée au VIH chez les sujets co-infectés. De surcroît, cer- taines observations préliminaires permettent de penser que la tuberculose peut accélérer la progression de l'infection par le VIH et qu'une chimiothérapie antituberculeuse préventive peut offrir la possibilité de prolonger la survie des sujets infectés par le VIH.

Ainsi, il peut être indiqué, à titre de précaution de santé individuelle, d'administrer de l'isoniazide à titre préventif contre la tuberculose chez un sujet séropositif pour le VIH qui est également positif au test à la tuberculine, si ce sujet ne présente pas de tuberculose évolutive. Pour administrer un traitement préventif en toute sécurité, il est impératif de disposer des équipements appropriés pour dépister les sujets présentant une tuberculose évolutive, et de suivre les patients pendant le traitement. Le dépistage doit comprendre au minimum une radiographie thoracique et un examen microscopique des bacilles acido-résistants. Il est également souhaitable de disposer des équipements nécessaires pour procéder à des cultures de mycobactéries prélevées dans les échantillons.

\section{Dépistage de la tuberculose et de la tuberculose-infection}

Tout service de conseil avant ou après un test de dépistage du VIH/SIDA devrait comporter une information sur la tuberculose et ses liens avec l'infection par le VIH. Les sujets trouvés séropositifs pour le VIH devraient être soumis à des examens cliniques de dépistage de la tuberculose. Ceux pour qui l'on envisage un traitement préventif de la tuberculose devraient recevoir une injection intradermique de tuberculine (épreuve de Mantoux) avec un produit équivalant à 5 UT de PPD-S ou de 2 UT de PPDRT23. Un test positif dénotant la présence d'une tuberculose-infection correspond par définition à une réaction consistant en une induration d'au moins $5 \mathrm{~mm}$ au bout de 48 à 72 heures. Bien que le BCG induise une réactivité au PPD, un seuil de $5 \mathrm{~mm}$ est approprié pour les adultes atteints d'une infection par le VIH, quelles que soient les vaccinations antérieures.

Il est de la plus haute importance d'exclure la présence de tuberculose évolutive avant de commencer un traitement préventif. Les sujets dont la réaction cutanée à la tuberculine est positive doivent donc subir une radiographie thoracique. Il faut également, chez ceux qui présentent des symptômes évocateurs de tuberculose pulmonaire et chez ceux dont la radiographie thoracique comporte des anomalies, procéder à des examens bactériologiques d'échantillons de crachats. Les patients qui présentent des signes et symptômes évocateurs de tuberculose extrapulmonaire (par exemple une adénopathie périphérique) doivent subir les examens appropriés. 
Les sujets séropositifs pour le VIH dont la réaction cutanée à la tuberculine est négative et qui vivent dans des régions d'endémie tuberculeuse peuvent être recontrôlés périodiquement (par exemple tous les 6 mois) et traités en conséquence. L'anergie à la tuberculine est fréquente chez les sujets infectés par le VIH, en particulier lorsque l'immunodépression est déjà avancée. Bien que l'on ne dispose que de peu de données à l'appui d'un traitement préventif par l'isoniazide des sujets anergiques exposés à un risque de tuberculose, ce traitement a été recommandé par certaines autorités. Toutefois, l'administration d'isoniazide à titre préventif à des sujets infectés par le VIH qui n'ont pas subi le test à la tuberculine n'a pas été évaluée et ne peut être actuellement recommandée.

\section{Administration d'isoniazide à titre préventif}

Le traitement préventif par l'isoniazide est indiqué chez les sujets infectés par le VIH et positifs au test à la tuberculine qui ne présentent pas de tuberculose évolutive. Le traitement préventif, aussi bien chez l'enfant que chez l'adulte, comprend l'administration d'une dose quotidienne d'isoniazide à raison de $5 \mathrm{mg} / \mathrm{kg}$ jusqu'à un maximum de $300 \mathrm{mg}$, pendant 6 à 12 mois. Les médicaments doivent être fournis tous les mois et les patients contrôlés pour vérifier l'observance du traitement, la toxicité du médicament et la présence de signes de tuberculose évolutive. L'isoniazide peut être administré 2 fois par semaine à raison de $15 \mathrm{mg} / \mathrm{kg}$ lorsqu'une surveillance est nécessaire, quoique aucune étude clinique n'ait démontré l'efficacité d'un traitement préventif intermittent. Les patients qui interrompent le traitement peuvent le reprendre afin que leur traitement préventif dure au moins 6 mois sur une période d'un an. Ces patients ne doivent néanmoins pas reprendre le traitement préventif s'ils manifestent des signes ou symptômes de tuberculose et si la présence d'une tuberculose évolutive n'a pas été exclue.

L'hépatotoxicité associée à l'isoniazide est le principal effet indésirable de ce médicament et peut apparaître chez $2 \%$ au maximum des sujets de plus de 50 ans. Le traitement préventif à l'isoniazide est contre-indiqué chez les sujets souffrant d'hépatite chronique active et doit être administré avec prudence en cas de consommation quotidienne d'alcool. Bien qu'il ne soit pas recommandé de surveiller en permanence l'apparition d'une hépatite au moyen d'examens biochimiques de routine, il faut mettre soigneusement les patients en garde contre la manifestation de signes ou symptômes d'hépatite et leur donner pour instruction d'arrêter rapidement la prise du médicament en présence de tels signes ou symptômes.

\section{Autres schémas thérapeutiques préventifs}

Certaines autorités ont recommandé l'application d'autres schémas thérapeutiques préventifs chez les sujets qui ne tolèrent pas l'isoniazide et chez ceux qui sont infectés par des organismes résistants à ce médicament; il existe peu de données cliniques et expérimentales pour étayer cette application. Toutefois, l'efficacité de ces schémas dans la prévention de la tuberculose chez les sujets infectés par le VIH n'a pas encore été démontrée, et aucun schéma thérapeutique de prévention n'est actuellement recommandé pour remplacer l'isoniazide.

\section{Recherche}

Seuls des travaux de recherche peuvent apporter une réponse à un certain nombre de questions concernant le traitement préventif de la tuberculose en présence d'une infection par le VIH. Au nombre des questions les plus importantes nécessitant des travaux de recherche appliquée, on peut citer l'efficacité des traitements de prévention polychimiothérapeutiques de brève durée, l'impact d'un traitement préventif prolongé ou administré la vie durant dans les zones à forte transmission de la tuberculose, et le rôle des traitements préventifs chez les sujets anergiques. Les études opérationnelles en vue de définir le rôle des traitements préventifs dans le contexte des programmes revêtent elles aussi la plus grande importance. Ces études consistent notamment à analyser avec soin la faisabilité des traitements préventifs en termes de demande, de durabilité et de rentabilité.

Une liste de références bibliographiques est disponible sur demande au Programme de la Tuberculose, Division des Maladies transmissibles, Organisation mondiale de la Santé, 1211 Genève 27, Suisse.

\section{Chimioprophylaxie du paludisme: schémas médicamenteux recommandés aux voyageurs ${ }^{c}$}

Les recommandations, pays par pays, sur les schémas médicamenteux pour la chimioprophylaxie du paludisme ont été révisées par l'OMS. On en trouvera ci-après un résumé, avec les zones dans lesquelles les différents schémas doivent être utilisés et les effets secondaires courants des médicaments.

\footnotetext{
c D'après: Chimioprophylaxie du paludisme: schémas médicamenteux recommandés aux voyageurs. Relevé épidémiologique hebdomadaire, 1993, 68(51): 377-383.
} 